\title{
MINIMUM ENERGY CONTROL OF 2D POSITIVE CONTINUOUS-DISCRETE LINEAR SYSTEMS
}

\author{
Tadeusz KACZOREK
}

${ }^{\star}$ Faculty of Electrical Engineering, Bialystok University of Technology, ul. Wiejska 45D, 15-351 Bialystok, Poland

kaczorek@isep.pw.edu.pl

\begin{abstract}
The minimum energy control problem for the 2D positive continuous-discrete linear systems is formulated and solved. Necessary and sufficient conditions for the reachability at the point of the systems are given. Sufficient conditions for the existence of solution to the problem are established. It is shown that if the system is reachable then there exists an optimal input that steers the state from zero boundary conditions to given final state and minimizing the performance index for only one step $(\boldsymbol{q}=\mathbf{1})$. A procedure for solving of the problem is proposed and illustrated by a numerical example.
\end{abstract}

Key words: 2D Continuous-Discrete, Linear, Positive, Reachability, Minimum Energy Control

\section{INTRODUCTION}

A dynamical system is called positive if its trajectory starting from any nonnegative initial state remains forever in the positive orthant for all nonnegative inputs. An overview of state of the art in positive theory is given in the monographs Farina and Rinaldi (2000) and Kaczorek (2001). Variety of models having positive behavior can be found in engineering, economics, social sciences, biology and medicine, etc.

The positive continuous-discrete 2D linear systems have been introduced in Kaczorek (1998), positive hybrid linear systems in Kaczorek (2007) and the positive fractional 2D hybrid systems in Kaczorek (2008a). Different methods of solvability of 2D hybrid linear systems have been discussed in Kaczorek et al. (2008) and the solution to singular 2D hybrid linear systems has been derived in Sajewski (2009). The realization problem for positive 2D hybrid systems has been addressed in Kaczorek (2008b). Some problems of dynamics and control of $2 \mathrm{D}$ hybrid systems have been considered in Dymkov et al. (2004) and Gałkowski et al. (2003). The problems of stability and robust stability of $2 \mathrm{D}$ continuous-discrete linear systems have been investigated in Bistritz (2003), Busłowicz (2010a, b), Narendra (2010), Sajewski $(2009)$ and Xiao $(2001,2003)$. Recently the stability and robust stability of general model and of Roesser type model of scalar continuous-discrete linear systems have been analyzed by Busłowicz (2010). New stability tests for positive standard and fractional linear systems have been proposed in Kaczorek (2011a). Stability of continuous-discrete 2D linear systems has been considered in Kaczorek (2011b).

The minimum energy control problem for standard linear systems has been formulated and solved by Klamka $(1976,1983$, 1991 ) and for 2D linear systems with variable coefficients in Kaczorek and Klamka (1986). The controllability and minimum energy control problem of fractional discrete-time linear systems has been investigated by Klamka (2010). The minimum energy control of fractional positive continuous-time linear systems has been addressed in Kaczorek $(2012,2014)$ and for descriptor positive discrete-time linear systems in Kaczorek (2013).

In this paper the minimum energy control problem for positive
2D continuous-discrete linear systems will be formulated and solved.

The paper is organized as follows. In section 2 the basic definitions and theorems of the positive continuous-discrete linear systems are recalled. Necessary and sufficient conditions for the reachability of the positive systems are given in section 3 . The minimum energy control problem is formulated and solved in section 4. A procedure for computation of the optimal inputs and the minimum value of the performance index are proposed and illustrated by numerical example in section 5 . Concluding remarks are given in section 6 .

The following notation will be used: $\Re$ - the set of real numbers, $\Re^{n \times m}$ - the set of $n \times m$ real matrices, $\mathfrak{R}_{+}^{n \times m}$ - the set of $n \times m$ matrices with nonnegative entries and $\Re_{+}^{n}=\Re_{+}^{n \times 1}$, $M_{n}$ - the set of $n \times n$ Metzler matrices (real matrices with nonnegative off-diagonal entries), $I_{n}$ - the $n \times n$ identity matrix.

\section{PRELIMINERIES}

Consider the general model of linear continuous-discrete system described by the equation:

$$
\begin{aligned}
& \dot{x}(t, i+1)=A_{0} x(t, i)+A_{1} \dot{x}(t, i)+A_{2} x(t, i+1) \\
& +B_{0} u(t, i)+B_{1} \dot{u}(t, i)+B_{2} u(t, i+1)
\end{aligned}
$$

where: $\dot{x}(t, i)=\frac{\partial x(t, i)}{\partial t}, x(t, i) \in \Re^{n}, u(t, i) \in \Re^{m}$ are the state and input vectors and $A_{k} \in \Re^{n \times n}, B_{k} \in \Re^{n \times m}$, $k=0,1,2$.

Boundary conditions for (1) are given by:

$$
\begin{aligned}
& x_{0 i}=x(0, i), i \in Z_{+}, \\
& x_{t 0}=x(t, 0), \dot{x}_{t 0}=\dot{x}(t, 0), t \in \Re_{+}
\end{aligned}
$$

The transition matrix of the model (1) is defined as follows:

$$
T_{i j}=\left\{\begin{array}{c}
I_{n}, \text { for } i=1=0 \\
A_{0} T_{i-1, j-1}+A_{1} T_{i, j-1}+A_{2} T_{i-1, j} \\
=T_{i-1, j-1} A_{0}+T_{i, j-1} A_{1}+T_{i-1, j} A_{2} \\
0, \text { for } i>0 \text { or } j<0
\end{array}\right.
$$


Theorem 1. The solution of the equation (1) with boundary conditions (2) has the form (proof is given in Kaczorek (2012)):

$$
\begin{aligned}
& x(t, i) \\
& =\sum_{k=0}^{\infty} \sum_{l=0}^{\infty}\left(T_{k, i-l-1} B_{0} \int_{0}^{t} \frac{(t-\tau)^{k}}{k !} u(\tau, l) d \tau\right. \\
& +T_{k, i-l} B_{2} \int_{0}^{t} \frac{(t-\tau)^{k}}{k !} u(\tau, l) d \tau \\
& -T_{k, i-l-1} B_{1} \frac{t^{k}}{k !} u(0, l)+T_{k, i-l} \frac{t^{k}}{k !} x(0, l) \\
& \left.-T_{k, i-l-1} A_{1} \frac{t^{k}}{k !} x(0, l)\right) \\
& +\sum_{k=1}^{\infty} \sum_{l=0}^{\infty}\left(T_{k, i-l-1} B_{1} \int_{0}^{t} \frac{(t-\tau)^{k-1}}{(k-1) !} u(\tau, l) d \tau\right) \\
& +\sum_{l=0}^{\infty} T_{0, i-l-1} B_{1} u(t, l) \\
& -\sum_{k=0}^{\infty}\left(T_{k, i} B_{2} \int_{0}^{t} \frac{(t-\tau)^{k}}{k !} u(\tau, 0) d \tau\right. \\
& \left.+T_{k, i} A_{2} \int_{0}^{t} \frac{(t-\tau)^{k}}{k !} x(\tau, 0) d \tau+T_{k, i} \frac{t^{k}}{k !} x(0,0)\right) \\
& +\sum_{k=1}^{\infty}\left(T_{k, i} \int_{0}^{t} \frac{(t-\tau)^{k-1}}{(k-1) !} x(\tau, 0) d \tau\right)+T_{0, i} x(t, 0)
\end{aligned}
$$

Definition 1. The general model (1) is called positive if $x(t, i) \in$ $\mathfrak{R}_{+}^{n}, t \in \mathfrak{R}_{+}, i \in Z_{+}$for any boundary conditions $x_{0 i} \in \mathfrak{R}_{+}^{n}$, $x_{t 0} \in \mathfrak{R}_{+}^{n}, \dot{x}_{t 0} \in \mathfrak{R}_{+}^{n}, t \in \mathfrak{R}_{+}$and all inputs $u(t, i) \in \mathfrak{R}_{+}^{m}$, $\dot{u}(t, i) \in \Re_{+}^{m}, t \in \Re_{+}, i \in Z_{+}$.

Theorem 2. The general model (2.1) is positive if and only if:

$$
\begin{aligned}
& A_{2} \in M_{n}, \quad A_{0}, A_{1} \in \mathfrak{R}_{+}^{n \times n} \\
& A_{0}+A_{1} A_{2} \in \mathfrak{R}_{+}^{n \times n}, B_{0}, B_{1}, B_{2} \in \Re_{+}^{n \times m}
\end{aligned}
$$

Proof is given in in Kaczorek (2012).

\section{REACHABILITY OF POSITIVE SYSTEMS}

Definition 2. The model (1) is called reachable at the point $\left(t_{f}, q\right)$ if for any given final state $x_{f} \in \mathfrak{R}^{n}$ there exists an input $u(t, i), 0 \leq t \leq t_{f}, 0 \leq i \leq q$ which steers the system form zero boundary conditions to the state $x_{f}$, i.e. $\left(t_{f}, q\right)=x_{f}$.

A square real matrix is called monomial if its every column and its every row has only one positive entry and the remaining entries are zero. The inverse of monomial matrix is also a monomial matrix (Kaczorek, 2012).

Theorem 3. The model (1) with $B_{1}=B_{2}=0$ is reachable at the point $\left(t_{f}, 1\right)$ if and only if the matrix $A_{2} \in M_{n}$ is diagonal and the matrix $B_{0} \in \mathfrak{R}_{+}^{n \times m}$ is monomial.

Proof. Sufficiency. If $A_{2} \in M_{n}$ is diagonal then $e^{A_{2} t}$ is also diagonal and if $B_{0} \in \mathfrak{R}_{+}^{n \times m}$ is monomial then $B_{0} B_{0}^{T}$ is also monomial. In this case the matrix:

$$
R_{f}=\int_{0}^{t_{f}} e^{A_{2} \tau} B_{0} B_{0}^{T} e^{A_{2}^{T} \tau} d \tau, \quad t_{f}>0
$$

is also monomial and $R_{f}^{-1} \in \Re_{+}^{n \times n}$.

The input:

$$
u(t)=u(t, 0)=B_{0}^{T} e^{A_{2}^{T}\left(t_{f}-\tau\right)} R_{f}^{-1} x_{f}
$$

steers the state of the system (1) from zero boundary conditions to $x_{f}$. The solution of (1) for $i=0, t=t_{f}$ has the form:

$$
x\left(t_{f}, 1\right)=\int_{0}^{t_{f}} e^{A_{2}\left(t_{f}-\tau\right)} B_{0} u(\tau) d \tau
$$

and substitution (7) onto (8) we obtain:

$$
x\left(t_{f}, 1\right)=\int_{0}^{t_{f}} e^{A_{2}\left(t_{f}-\tau\right)} B_{0} B_{0}^{T} e^{A_{2}^{T}\left(t_{f}-\tau\right)} d \tau R_{f}^{-1} x_{f}=x_{f}
$$

The necessity can be proved in a similar way as in Kaczorek (2012, 2013a).

\section{MINIMUM ENERGY CONTROL PROBLEM FOR POSITIVE SYSTEMS WITH BOUNDED INPUTS}

\subsection{Problem formulation}

Consider the positive 2D continuous-discrete system (1) with $A_{2} \in M_{n}$ and $B_{0} \in \mathfrak{R}_{+}^{n \times m}$ monomial and $B_{1}=B_{2}=0$. If the system is reachable in time $t \in\left[0, t_{f}\right]$, then usually there exists many different inputs $u(t) \in \mathfrak{R}_{+}^{n}$ that steers the state of the system from zero boundary conditions to $x_{f} \in \mathfrak{R}_{+}^{n}$. Among these inputs we are looking for an input $u(t) \in \mathfrak{R}_{+}^{n}$ that minimizes the performance index:

$$
I(u)=\int_{0}^{t_{f}} u^{T}(\tau) Q u(\tau) d \tau
$$

where: $\mathcal{Q} \in \mathfrak{R}_{+}^{n \times n}$ is a symmetric positive defined matrix and $\mathcal{Q}^{-1} \in \mathfrak{R}_{+}^{n \times n}$.

The minimum energy control problem for the positive 2D continuous-discrete linear systems (1) can be stated as follows: Given the matrices: diagonal $A_{2} \in M_{n}$, monomial $B_{0} \in \mathfrak{R}_{+}^{n \times n}, t_{f}$, $q=1$ and $\mathcal{Q} \in \mathfrak{R}_{+}^{n \times n}$ of the performance index (10), $x_{f} \in \mathfrak{R}_{+}^{n}$ and $t_{f}>0$, find an input $u(t) \in \Re_{+}^{n}$ for $t \in\left[0, t_{f}\right]$ that steers the state vector of the system from zero boundary conditions to $x_{f} \in \mathfrak{R}_{+}^{n}$ and minimizes the performance index (10).

\subsection{Problem solution}

To solve the problem we define the matrix:

$$
W=W\left(t_{f}, \mathcal{Q}\right)=\int_{0}^{t_{f}} e^{A_{2}\left(t_{f}-\tau\right)} B_{0} \mathcal{Q}^{-1} B_{0}^{T} e^{A_{2}^{T}\left(t_{f}-\tau\right)} d \tau
$$

From (11) and Theorem 3 it follows that the matrix (11) is monomial if and only if the positive system (1) is reachable at the point $\left[t_{f}, 1\right]$. In this case we may define the input:

$$
\widehat{u}(t)=\mathcal{Q}^{-1} B_{0}^{T} e^{A^{T}\left(t_{f}-\tau\right)} W^{-1} x_{f} \text { for } t \in\left[0, t_{f}\right]
$$

Note that the input (12) satisfies the condition $\hat{u}(t) \in \mathfrak{R}_{+}^{n}$ for $t \in\left[0, t_{f}\right]$ if:

$$
\mathcal{Q}^{-1} \in \mathfrak{R}_{+}^{n \times n} \text { and } W^{-1} \in \mathfrak{R}_{+}^{n \times n}
$$


Theorem 4. Let the positive system (1) be reachable at the point $\left[t_{f}, 1\right]$ and let $\bar{u}(t) \in \Re_{+}^{n}$ for $t \in\left[0, t_{f}\right]$ be an input that steers the state of the positive system (1) from zero boundary conditions to $x_{f} \in \mathfrak{R}_{+}^{n}$. Then the input (12) also steers the state of the system from zero boundary conditions to $x_{f} \in \mathfrak{R}_{+}^{n}$ and minimizes the performance index (10), i.e. $I(\hat{u}) \leq I(\bar{u})$. The minimal value of the performance index (10) is equal to:

$$
I(\hat{u})=x_{f}^{T} W^{-1} x_{f}
$$

Proof. If the conditions (13) are met then the input (12) is well defined and $\hat{u}(t) \in \mathfrak{R}_{+}^{n}$ for $t \in\left[0, t_{f}\right]$. We shall show that the input steers the state of the system from zero boundary conditions to $x_{f} \in \mathfrak{R}_{+}^{n}$. Substitution of (12) into (8) for $t=t_{f}$ and zero boundary conditions yields:

$$
\begin{aligned}
& x\left(t_{f}, 1\right)=\int_{0}^{t_{f}} e^{A_{2}\left(t_{f}-\tau\right)} B_{0} \hat{u}(\tau) d \tau \\
& =\int_{0}^{t_{f}} e^{A_{2}\left(t_{f}-\tau\right)} B_{0} Q^{-1} B_{0}^{T} e^{A_{2}^{T}\left(t_{f}-\tau\right)} d \tau W^{-1} x_{f}=x_{f}
\end{aligned}
$$

since (11) holds. By assumption the inputs $\bar{u}(t)$ and $\hat{u}(t), t \in$ $\left[0, t_{f}\right]$ steers the state of the system from zero boundary conditions to $x_{f} \in \Re_{+}^{n}$. Hence:

$$
\begin{aligned}
x_{f}=\int_{0}^{t_{f}} e^{A_{2}\left(t_{f}-\tau\right)} & B_{0} \bar{u}(\tau) d \tau \\
= & \int_{0}^{t_{f}} e^{A_{2}\left(t_{f}-\tau\right)} B_{0} \hat{u}(\tau) d \tau
\end{aligned}
$$

or:

$$
\int_{0}^{t_{f}} e^{A_{2}\left(t_{f}-\tau\right)} B_{0}[\bar{u}(\tau)-\hat{u}(\tau)] d \tau=0
$$

By transposition of (16b) and postmultiplication by $W^{-1} x_{f}$ we obtain:

$$
\int_{0}^{t_{f}}[\bar{u}(\tau)-\hat{u}(\tau)]^{T} B_{0}^{T} e^{A_{2}^{T}\left(t_{f}-\tau\right)} d \tau W^{-1} x_{f}=0
$$

Substitution of (12) into (17) yields:

$$
\begin{aligned}
& \int_{0}^{t_{f}}[\bar{u}(\tau)-\hat{u}(\tau)]^{T} B_{0}^{T} e^{A_{2}^{T}\left(t_{f}-\tau\right)} d \tau W^{-1} x_{f} \\
& =\int_{0}^{t_{f}}[\bar{u}(\tau)-\hat{u}(\tau)]^{T} \mathcal{Q}(\tau) d \tau=0
\end{aligned}
$$

Using (18) it is easy to verify that:

$$
\begin{aligned}
\int_{0}^{t_{f}} \bar{u}(\tau)^{T} \mathcal{Q u}(\tau) d \tau=\int_{0}^{t_{f}} \hat{u}(\tau)^{T} \mathcal{Q} \hat{u}(\tau) d \tau \\
\quad+\int_{0}^{t_{f}}[\bar{u}(\tau)-\hat{u}(\tau)]^{T} \mathcal{Q}[\bar{u}(\tau)-\hat{u}(\tau)] d \tau
\end{aligned}
$$

From (19) it follows that $I(\hat{u}) \leq I(\bar{u})$ since the second term in the right-hand side of the inequality is nonnegative.

To find the minimal value of the performance index (10) we substitute (12) into (10) and we obtain:

$$
\begin{aligned}
& I(\hat{u})=\int_{0}^{t_{f}} \hat{u}^{T}(\tau) \mathcal{Q} \hat{u}(\tau) d \tau \\
& =x_{f}^{T} W^{-1} \int_{0}^{t_{f}} e^{A_{2}\left(t_{f}-\tau\right)} B_{0} Q^{-1} B_{0}^{T} e^{A_{2}^{T}\left(t_{f}-\tau\right)} d \tau W^{-1} \lambda \\
& =x_{f}^{T} W^{-1} x_{f}
\end{aligned}
$$

since (11) holds.

We have the following two important conclusions:

Conclusion 1. If the matrix $A_{2} \in M_{n}$ is diagonal and the matrix $B_{0} \in \mathfrak{R}_{+}^{n \times m}$ is monomial then there exists an input (7) that steers the state of the positive system (1) from zero boundary conditions to $x_{f} \in \mathfrak{R}_{+}^{n}$ and minimize the performance index (10).

Conclusion 2. For $q=1$ the optimal input (7) and the minimal value of the performance index (14) are independent of the matrices $A_{0}$ and $A_{1}$ of the positive system (1).

The considerations can be easily extended to $q>1$.

\section{PROCEDURE AND EXAMPLE}

From the considerations of section 4 we have the following procedure for computation the optimal inputs that steers the state of the system from zero boundary conditions to $x_{f} \in \mathfrak{R}_{+}^{n}$ and minimize the performance index (10).

Procedure 1.

Step 1. Knowing $A_{2} \in M_{n}$ compute $e^{A_{2} t}$.

Step 2. Knowing the matrices $A_{2}, B_{0}, Q$ for some $t_{f}$ and using (11) compute the matrix $W$.

Step 3. Using (12) compute $\hat{u}(\tau)$.

Step 4. Using (10) compute the minimal value of the performance index $I(\hat{u})$.

Example 1. Consider the positive system (1) with matrices:

$A_{2}=\left[\begin{array}{cc}-1 & 0 \\ 0 & -2\end{array}\right], \quad B_{0}=\left[\begin{array}{ll}0 & 1 \\ 1 & 0\end{array}\right]$

and the performance index (10) with:

$\mathcal{Q}=\left[\begin{array}{ll}2 & 0 \\ 0 & 2\end{array}\right]$

Compute the optimal input $\hat{u}(t)$ for $t \in[0,1]$ and $q=1$ that steers the state of the system from zero boundary conditions to $x_{f}=\left[\begin{array}{ll}1 & 1\end{array}\right]^{T} \in \Re_{+}^{2} \quad(T$ denote the transpose $)$ and minimize the performance index.

By Theorem 3 the positive system (1) with (21) is reachable at the point $\left(t_{f}=1, q=1\right)$. Using the Procedure 1 we obtain the following:

Step 1. In this case we have:

$e^{A_{2} t}=\left[\begin{array}{cc}e^{-t} & 0 \\ 0 & e^{-2 t}\end{array}\right]$

Step 2. Using (11), (21) and (23) we obtain:

$W=\int_{0}^{t_{f}} e^{A_{2}\left(t_{f}-\tau\right)} B_{0} \mathcal{Q}^{-1} B_{0}^{T} e^{A_{2}^{T}\left(t_{f}-\tau\right)} d \tau$

$=\int_{0}^{t_{f}}\left[\begin{array}{cc}e^{-2 \tau} & 0 \\ 0 & e^{-4 \tau}\end{array}\right]=\left[\begin{array}{cc}\frac{1}{4}\left(1-e^{-2}\right) & 0 \\ 0 & \frac{1}{8}\left(1-e^{-4}\right)\end{array}\right]$ 
Step 3. Using (12) and (22)-(24) we obtain:

$$
\begin{aligned}
& \hat{u}=\mathcal{Q}^{-1} B_{0}^{T} e^{A_{2}^{T}\left(t_{f}-\tau\right)} W^{-1} x_{f} \\
& =\frac{1}{2}\left[\begin{array}{cc}
0 & e^{2(t-1)} \\
e^{(t-1)} & 0
\end{array}\right]\left[\begin{array}{cc}
\frac{1}{4}\left(1-e^{-2}\right) & 0 \\
0 & \frac{1}{8}\left(1-e^{-4}\right)
\end{array}\right]^{-1}\left[\begin{array}{l}
1 \\
1
\end{array}\right] \\
& =\left[\begin{array}{l}
\frac{4 e^{2(t-1)}}{\left(1-e^{-4}\right)} \\
\frac{2 e^{(t-1)}}{\left(1-e^{-2}\right)}
\end{array}\right]
\end{aligned}
$$

Step 4. The minimal value of the performance index is equal to:

$$
\begin{aligned}
& I(\hat{u})=x_{f}^{T} W^{-1} x_{f} \\
& =\left[\begin{array}{ll}
1 & 1
\end{array}\right]\left[\begin{array}{cc}
\frac{1}{4}\left(1-e^{-2}\right) & 0 \\
0 & \frac{1}{8}\left(1-e^{-4}\right)
\end{array}\right]^{-1}\left[\begin{array}{l}
1 \\
1
\end{array}\right] \\
& =\frac{4}{\left(1-e^{-2}\right)}+\frac{8}{\left(1-e^{-4}\right)}
\end{aligned}
$$

\section{CONCLUDING REMARKS}

The minimum energy control problem for the 2D positive continuous-discrete linear systems has been formulated and solved. Necessary and sufficient conditions for the reachability at the point $\left(t_{f}, q=1\right)$ of the systems have been established (Theorem 3$)$. Sufficient conditions for the existence of solution to the minimum energy control problem of the positive 2D continuous-discrete linear systems have been given (Theorem 4). The procedure for computation of the optimal input and the minimal value of performance index has been proposed and illustrated by numerical example. It has been shown that if the system is reachable then there exists an optimal input that steers the state from zero boundary conditions to given final state and minimizing the performance index for only one step for $q=1$. The consideration can be easily extended to $q>1$ and for (1) with nonzero matrices $B_{k}, k=0,1,2$. In the subsequent paper the considerations will be extended to the descriptor positive 2D continuous-discrete linear systems.

\section{REFERENCES}

1. Bistritz Y. (2003), A stability test for continuous-discrete bivariate polynomials, Proc. Int. Symp. on Circuits and Systems, Vol. 3, 682-685.

2. Busłowicz M. (2010), Robust stability of the new general $2 \mathrm{D}$ model of a class of continuous-discrete linear systems, Bull. Pol. Acad. Sci. Techn., Vol. 57, No. 4, 561-565.

3. Busłowicz M. (2010), Stability and robust stability conditions for a general model of scalar continuous-discrete linear systems, Measurement Automation and Monitoring, Vol. 56, No. 2, 133-135.

4. Dymkov M., Gaishun I., Rogers E., Gałkowski K. and Owens D.H. (2004), Control theory for a class of 2D continuous-discrete linear systems, Int. J. Control, Vol. 77, No. 9, 847-860.
5. Farina L. and Rinaldi S. (2000), Positive Linear Systems; Theory and Applications, J. Wiley, New York.

6. Gałkowski K., Rogers E., Paszke W. and Owens D.H. (2003), Linear repetitive process control theory applied to a physical example, Int. J. Appl. Math. Comput. Sci., Vol. 13, No. 1, 87-99.

7. Kaczorek T. (1998), Reachability and minimum energy control of positive 2D continuous-discrete systems, Bull. Pol. Acad. Sci. Tech., Vol. 46, No. 1, 85-93.

8. Kaczorek T. (2001), Positive $1 D$ and $2 D$ systems, Springer Verlag, London.

9. Kaczorek T. (2007), Positive 2D hybrid linear systems, Bull. Pol. Acad. Sci. Tech., Vol. 55, No. 4, 351-358.

10. Kaczorek T. (2008a), Positive fractional 2D hybrid linear systems, Bull. Pol. Acad. Tech., Vol. 56, No. 3, 273-277.

11. Kaczorek T. (2008b), Realization problem for positive 2D hybrid systems, COMPEL, Vol. 27, No. 3, 613-623.

12. Kaczorek T. (2011a), New stability tests of positive standard and fractional linear systems, Circuit and Systems, Vol. 2, No. 4, 261-268.

13. Kaczorek T. (2011b), Stability of continuous-discrete linear systems described by general model, Bull. Pol. Acad. Sci. Tech., Vol. 59, No. 2, 189-193.

14. Kaczorek T. (2012), Selected Problems of Fractional Systems Theory, Springer-Verlag, Berlin.

15. Kaczorek T. (2013), Minimum energy control of fractional positive continuous-time linear systems, Proc. of Conf. MMAR, Międzyzdroje, Poland.

16. Kaczorek T. (2013), Minimum energy control of positive fractional descriptor continous time linear systems, IET Control Theory and Applications, Vol. 8 No. 4, 219-225.

17. Kaczorek T. (2014), Minimum energy control of descriptor positive discrete-time linear systems, Compel, Vol. 33, No.3, 1-14.

18. Kaczorek T., Klamka J. (1986), Minimum energy control of 2D linear systems with variable coefficients, Int. J. of Control, Vol. 44, No. 3, 645-650.

19. Kaczorek T., Marchenko V. and Sajewski Ł. (2008), Solvability of 2D hybrid linear systems - comparison of three different methods, Acta Mechanica et Automatica, Vol. 2, No. 2, 59-66.

20. Klamka J. (1976), Relative controllability and minimum energy control of linear systems with distributed delays in control, IEEE Trans. Autom. Contr., Vol. 21, No. 4, 594-595.

21. Klamka J. (1983), Minimum energy control of 2D systems in Hilbert spaces, System Sciences, Vol. 9, No. 1-2, 33-42.

22. Klamka J. (1991), Controllability of Dynamical Systems, Kluwer Academic Press, Dordrecht.

23. Klamka J. (2010), Controllability and minimum energy control problem of fractional discrete-time systems, Chapter in New Trends in Nanotechnology and Fractional Calculus, Eds. Baleanu D., Guvenc Z.B., Tenreiro Machado J.A., Springer-Verlag, New York, 503-509.

24. Narendra K.S. and Shorten R. (2010), Hurwitz stability of Metzler matrices, IEEE Trans. Autom. Contr., Vol. 55, No. 6, 1484-1487.

25. Sajewski $Ł$. (2009), Solution of 2D singular hybrid linear systems, Kybernetes, Vol. 38, No. 7/8, 1079-1092.

26. Xiao Y. (2001), Stability test for 2-D continuous-discrete systems, Proc. 40th IEEE Conf. on Decision and Control, Vol. 4, 3649-3654.

27. Xiao Y. (2003), Stability, controllability and observability of 2-D continuous-discrete systems, Proc. Int. Symp. on Circuits and Systems, Vol. 4, 468-471.

Acknowledgment: This work was supported under work S/WE/1/11. 